\title{
Research on Promotion of Modern Media and Undergraduate Growth in College Communities
}

\author{
Rongfang Li \\ Oxbridge College, Kunming University of Science and Technology, Kunming, \\ 650106, China
}

\begin{abstract}
Modern media have already become an important platform for contemporary college students to obtain knowledge, enrich life and communicate \& exchange. With continuous development of modern media, it has more and more influence on studies and life of college students. Moreover, modern media also constantly change values, views of life and world views of contemporary undergraduates. On this basis, the author discussed in this paper the promotion of modern media and growth of undergraduates, and analyzed positive and negative effects of modern media, with expectations to have certain reference and help for growth of contemporary college students.
\end{abstract}

Key words: college community, promotion of modern media, undergraduate growth

The development and popularization of modern media technology has wide and profound influence on mode of thinking, behavior pattern and values of contemporary college students. At the same time, it also expands new channels and means for enhancing and perfecting ideological and political education in colleges and universities. We should combine behavior characteristics of undergraduates contacting new media to research and explore for the interactive mechanism of the promotion of community modern media and cultivation of growth of undergraduates, utilize technical features of modern media, combine self education of college students and promote wide and deep interaction between education subjects.

With the development of the internet technology, China also entered the Internet era. Under such background, all kinds of modern media also got rapid development, such as microblog, WeChat and other medium forms. Moreover, continuous popularization of mobile intelligent terminal in recent years also promoted the increasing number of Chinese college students to using modern media. Contemporary college students become resources with the highest utilization of modern media. Therefore, modern media have deeper and deeper influence on 
contemporary college students.

\section{Positive Effects of Modern Media on Undergraduate Growth}

\section{Important channels for undergraduates to obtain knowledge}

With the development of global informatization and networking, the Internet is featured by large amount of information and fast propagation speed. It can effectively promote inheritance of Chinese culture and transmit more cultural knowledge and scientific culture through modern media. Moreover, the Internet broke propagation mode and approach of traditional knowledge, changed the limitation of giving more knowledge to students only through classroom teaching, enriched the contents of knowledge of contemporary college students, cultivated the exploration spirit and learning interest of undergraduates for fresh things and allows contemporary college students prefer to obtain more unknown information and knowledge through modern media, so as to improve professional skills and learning initiative of college students.

\section{Effective modes for communication and exchange of undergraduates}

The current Internet mobile terminal equipment has become an important communication platform in the present society. People can contact and interact through WeChat, microblog, QQ and other mobile software. At the same time, it also becomes an integral part of undergraduates' social activities. Undergraduates used many modern media to realize social communication between people and people and form effective interaction with students and teachers. Modern media broke limitations of time and space, which can be used to jointly discuss interesting topics with others. Moreover, modern media can be used to fully display ourselves and realize self-worth. Convenience and equality of modern media can be used to relieve stress of undergraduates, eliminate some unhealthy emotions, and improve social ability of undergraduates, thus radically meeting life and learning needs of contemporary college students.

\section{New approaches for ideological and political education of undergraduates}

To some extent, modern media have already deeply influenced life and studies of contemporary college students as well as their views and values. Therefore, modern media became a new platform and a new challenge for ideological and political education of college students. Moreover, modern media changed traditional single educational pattern, broke the limitations of time and space, and enhanced interactivity between students. Modern media have already gradually become a new 
platform for issuing information, guiding public opinions and educating ideology and politics in the present college campus. Now, many schools have already established microblog, official public WeChat and other forms to develop ideological and political education, letting numerous students become their loyal fans. Interactivity of modern media is used to have an all-round understanding of students' thought. Students are correctly guided through interaction and communication. Moreover, contemporary college students are helped to set up correct views and values, effectively promoting integrated development of undergraduates.

\section{An important platform for expanding undergraduates' cultural life}

With continuous development of modern media, people's social activity fields and abilities are continuously improved. Modern media have huge effects on spiritual and cultural life of present people. Due to its feature of large amount of information, modern media become an important platform for contemporary college students to obtain and enrich cultural life. Many cultural products are transmitted by modern media, supplementing cultural knowledge of undergraduates, and becoming an important approach used to enrich cultural life of college students and provide mental foraging. Modern media provided a platform and environment for contemporary college students to perfect and breakthrough themselves.

\section{Helpful for undergraduates to perform social positioning in a faster way}

The purpose of college education is to be able to quickly adapt to social activities through learning of knowledge and culture in the period of college and realize joint development of ego and society after entering social production activities. This is also a positioning process of social role. Through college education, students are made to become qualified excellent talents to transform and adapt to the society. Modern media can be used to allow students to receive more views and values, learn more social experiences and enhance social understandings of college students. Under the influence and promotion of modern media, it is beneficial for undergraduates to quickly perform social positioning, find their social roles and finish the socialization process at faster speed.

\section{Negative Effects of Modern Media on Undergraduate Growth}

1. Excessive dependence on modern media will bring forth frustrating effects on growth.

So-called excessive dependence refers to excessive addiction to modern media and 
the failure to make self adjustment or even extricate. All values and behavior activities need to find out answers through modern media. Thus, it will be easy to lead students to excessively live in the virtual environment, be unwilling to participate into more activities in real life and form the unsociable and eccentric character. Originally, students in the college period are immature in terms of will. No correct value or view is formed. In the rapidly developing modern network environment, it is easy for them to be addicted in that in the face of various temptations. For instance, some students are addicted into online games. They excessively used modern media to express themselves, unable to get rid of the virtual world. These students are made to become indifferent in the real life. More seriously, interpersonal relationship crisis even occurred. They thought that they could find recognition and express their self value in a better way only in the virtual world, resulting in frustrating consequences for their growth.

\section{Bad information in modern media will influence physical and psychological health of undergraduates.}

Modern media are of certain openness. In the entire promotion process in college communities, some bad information will appear. The bad information will easily let college students form vulgar cultural consciousness. Specific presentations of these phenomena can be directly reflected through some labels of modern media, such as good-luck face of women with big faces, jailbreak pig and god. All these are hot topics discussed by college students all the time. This also presents entertainment and non-mainstream of modern media, with adverse effects on physical and psychological health of undergraduates.

\section{Excessive attention on a large amount of information will influence the}

\section{learning time.}

The current modern media transmit a large amount of various kinds of information all day. Modern groups have already been submerged by considerable information. Although modern media brought forth new approaches for people to obtain information, such considerable information and so-called fragment information are also of certain adverse effects. It can be seen from the most popular WeChat. Public information in the circle of friends is constantly renewed everyday. Lots of copied and forwarded information even appeared. This fragment culture lets some students be addicted in that and have certain conscious misunderstanding. They thought that was a kind of reading and learning mode in modern media. Everyday, they spend 
much time in these contents. Moreover, most information is meaningless, seriously influencing thought and reflection of students, having no great help for their growth and studies.

\section{Openness of modern media caused behavior anomie of students.}

Here, behavior anomie refers to some behaviors violating self-discipline or morality in the use process of network. Network violation of laws, network crime and other contents are covered. Modern media provide an open platform. In this platform, students can release some irresponsible comments in modern media in the virtual and anonymous form. In the platform of modern media, undergraduates play certain virtual roles and lack certain moral and legal restraints. Even illegal behaviors will occur, such as creating and spreading rumors. In the new platform of modern media, some college students failed to form enough legal consciousness. Although it is a virtual platform, this platform is also a kind of extension of real life. Modern media are both private space and public space. Comments issued in this public space need to be legally restrained.

\section{Modern media should be correctly used to promote undergraduate growth.}

\section{Enhance self-improvement to media literacy of college students}

With the development of modern media, enhancing media literacy of college students becomes the primary focus. It is recommended to introduce relevant teaching contents into classroom education. Media literacy of college students should be cultivated from the following aspects: firstly, we should give correct guidance, enhance legal consciousness of contemporary college students and improve the use specifications in modern media against diversified concepts in modern media. Secondly, we should enhance the right and wrong resolving ability of contemporary college students. Contemporary college students should have certain ability of judgment for bad information, junk information and vulgar information in modern media, so as to boycott illegal and harmful information. Thirdly, we should correctly guide contemporary college students to use modern media, improve the efficiency and practicability and reach the purpose of self-improvement through the use of modern media.

\section{Strengthen education for values of college students}

For various kinds of bad information in modern media, we should strengthen guidance to values of undergraduates and give correct guidance by adhering to socialist core values and Marxist philosophy, so as to form unified values and 
cultural concepts among college students. Moreover, we should also strengthen spiritual culture education for students, laying a solid foundation for their social works. Relevant departments should strengthen the purification of modern media, establish modern media platforms helpful for physical and psychological health of college students, form equal interaction between the platform and students, timely master their thoughts, and assist them in setting up correct values, views of life and world views, maintaining positive attitudes, putting into studies of professional knowledge, enhancing mutual trust and understanding, eliminating contrasting feelings and forming wide consensus, so as to enhance the sense of belonging and collectivism spirit of teachers, make organizations have more cohesive force and make them to become high-quality talents adaptive to demands of social development as soon as possible.

\section{Intensify usage specifications for modern media}

Some cases can be used to educate college students for scandalized use of modern media, letting college students enhance self-requirement and consciousness and standardize their use specifications. Students should have a clear understanding that medium is also a kind of extension of real life, although it is a virtual platform. In real life, we need to follow laws and regulations. As extension of real life, modern media should also follow relevant laws, regulations and social morals. We should enhance media literacy of undergraduates and cultivate their legal consciousness by holding lectures on campus, so as to effectively improve their sense of social responsibility, promote the self-requirement consciousness of contemporary college students, and effectively avoid the occurrence of behavior anomie.

\section{Enhance correct modern media values of college students}

Whether the view of modern media is correct or not is a key factor for growth of undergraduates in the environment of modern media. We should establish relevant judgment consciousness among college students, letting them judge information appeared in modern media. Contemporary college students should be made to have a clear understanding of the essence of modern media, treat modern media with correct concepts and promote healthy growth of undergraduates with correct concepts. At the same time, we should correctly guide college students to transform information appeared in modern media. If not transformed, the information will become useless. Some information will even misguide us. Moreover, we should lead students to transform non-knowledge information into knowledge. In this 
process, it is necessary for students to have careful reflection and judgment, not only verifying the truth of information but also having certain cognitive competence, ability of judgment and theoretical duty, so as to effectively transform information in modern media into the contents of knowledge capable of guiding people's thoughts.

\section{Conclusion}

Modern media are of very important promotion for growth of contemporary college students. At the same time, there are also some negative effects. In short, we should use modern media with correct concepts, give play to the maximum advantages of modern media, give timely education and guidance against probable negative effects and let modern media become a new platform for college students to obtain knowledge, exchange thoughts as well as learning and growth.

\section{Acknowledgments}

This paper is the scientific research project of the teaching reform of the College in 2012: project name: Research on Promotion of Modern Media and Undergraduate Growth in College Communities (No.: JQJG201211).

\section{References:}

[1] Wang Di, New Challenges of New Media on Ideological and Political Education of Post-90s Undergraduates [J], Studies in Ideological Education, 2010, (01);

[2] James M. Henslin, Sociology: A Down-To-Earth Approach [M], Beijing: Peking University Press, 2007;

[3] Qian Wenbin, Impact of New Media on Undergraduates Value-orientation and Countermeasures [J], Press Circles, 2010, (03);

[4] Wu Caihong and Quan Chengxiang, Control over Network Behavior Anomie of Undergraduates [J], Journal of Hunan Financial and Economic College, 2008, (10); [5] Zhou Zhongxiang, Li Zhenwei and Wang Xuepeng, Survey and Reflection on Value Orientation o Post-90s Undergraduates [J], Journal of Inner Mongolia Agricultural University (Social Science Edition), 2012 (05);

[6] Jiang Xiaoli, Dong Ziming and Cao Yina, On the Interactive Mechanism of the Core Values for the College Students under the Training of New Media [J], Journal of Xiangtan University (Philosophy and Social Sciences), 2010 (05) 